\title{
Theoretical Study on Radial Distribution Laws of Rock Mass Damage Factors under Decoupled Charge Blasting
}

\author{
Qiang Pan, ${ }^{1}$ Jichun Zhang, ${ }^{1}$ Shuangying Zheng $\mathbb{D}^{1},{ }^{1}$ Hongchao Shi, ${ }^{2}$ and Zhanfeng Fan ${ }^{1}$ \\ ${ }^{1}$ School of Civil Engineering, Southwest Jiaotong University, Chengdu 610031, China \\ ${ }^{2}$ College of Architectural and Environmental Engineering, Chengdu Technological University, Chengdu 611730, China
}

Correspondence should be addressed to Shuangying Zheng; zsy2012@swjtu.edu.cn

Received 17 September 2019; Revised 10 June 2020; Accepted 3 July 2020; Published 28 July 2020

Academic Editor: Franck Poisson

Copyright (c) 2020 Qiang Pan et al. This is an open access article distributed under the Creative Commons Attribution License, which permits unrestricted use, distribution, and reproduction in any medium, provided the original work is properly cited.

In this paper, the radial distribution laws of damage factors under decoupled charge blasting are studied for the optimization design of blasting parameters. Through defining the critical radial decoupling coefficient, the damage zone around the borehole is partitioned and the characteristics are described. Based on the damage factor defined by Taylor's effective elastic modulus, the formulas of the radial distribution laws of damage factors are derived by the attenuation law of stress wave and the theory of thickwalled cylinder, respectively, which are then superposed to obtain the formula under the combined action of explosion stress wave and quasistatic gas. Experimental verification indicates that the theoretical values, which have a good correlation with the test data and are of high accuracy, can characterize the radial distribution laws of damage factors and estimate the damage range. When a radial decoupling coefficient is less than the critical value, the attenuation rate of damage factors firstly increases and then decreases with the increase of distance, and a serious damage zone is caused. Conversely, it decreases gradually, and the serious damage zone is not caused. Therefore, on the premise of stable detonation, it is necessary to apply an appropriate radial decoupling coefficient which is larger than the critical value to smooth or presplit blasting.

\section{Introduction}

During the process of formation and evolution of rock mass, there are a lot of initial damages such as microcracks and microcavities in the interior $[1,2]$. Microcracks are activated, grown, and coalesced gradually to form macrocracks under the load, which finally causes the physical and mechanical properties of rock mass to be lowered and even be failed [3]. Hence, rock mass failure is a process of the accumulation and evolution of damage [4]. Blasting action can inevitably cause different degrees and ranges of damage to the reserved rock mass. Due to the irreversible process of damage, the gradual accumulation and evolution cause rock mass to be fractured, which endangers the integrity and stability of rock mass. Therefore, the study on blasting damage is always one of the primary research topics in the field of rock engineering [5], especially damage control in the bedrock blasting excavation of an underground radioactive waste repository and a nuclear power plant $[6,7]$.
At present, studies on blasting damage in rock mass have mainly focused on constitutive models [4, 8-10], evolution mechanisms and characteristics [11-14], tests and safety assessments [15-19], control technologies [20-23], and so on. Among them, the damage range and degree are two extremely important contents of damage characteristics. However, up to now, relatively few studies have been done on the radial distribution laws of the damage factors within the blasting damage range. Carrying out the study can not only identify the radial distribution laws of damage factors and the interrelation between damage factors and blasting parameters but also estimate the damage range and further control damage.

Some scholars have studied the radial distribution laws of the blasting damage factors. Dai et al. [24] found that the damage factors around a single hole decreased in an approximate power function law with the increase of distance and fitted a relation between damage factors and scaled distance through the experimental data. Suo [25], through 
ultradynamic strain tests of coal samples, put forward that the volumetric strain accorded with the attenuation law of a power function and applied Taylor's and Grady's damage models to derive the distribution function of the macroscopic damage degree generated by preweakening the blasting of coal seams. Tang et al. [26] derived the distribution laws of damage factors based on the relation that elastic modulus was proportional to transmission frequency of stress wave as well as the relation of blasting dominant frequency to charge mass and to the distance from the explosion center. Meanwhile, five damage zones were partitioned. Pan et al. [27] found that damage factors presented an attenuation law of exponential function with distance and fitted out related parameters through numerical simulation. Although the abovementioned research achievements enrich the theory of blasting damage to some extent, there are the following shortages to be further improved. Empirical formulas fitted out by model experiments and numerical experiments still need theoretical supports. The existing theoretical analysis only considers the contributions of explosion stress wave to damage evolution, without considering the contributions of the quasistatic gas. When the strain analysis is applied, only the volumetric strain is considered, and the equivalent strain is ignored. Therefore, it is necessary to conduct a theoretical analysis on the radial distribution laws of damage factors under the combined action of explosion stress wave and quasistatic gas, which can provide a theoretical guidance for optimizing blasting parameters and carrying out model experiments and numerical experiments.

Based on the effective elastic modulus of mesodamage mechanics, the radial distribution laws of damage factors under the combined action of explosion stress wave and quasistatic gas are derived by the attenuation law of explosion stress wave and the change law of elastic stress field in the thick-walled cylinder. The correctness and reliability is validated by experimental data so as to lay a theoretical foundation for the optimization design of blasting parameters.

\section{Definition of Damage Factor and Partition of Damage Zone}

2.1. Definition of Damage Factor. At present, the definition of damage factor has not had a unified form. Applying macroscopic material parameters to characterize damage degree has been the common method. According to macroscopic material parameters, the damage factor of rock mass, $D$, can be expressed as

$$
D=1-\frac{\bar{E}}{E}=1-\left(\frac{\bar{c}_{p}}{c_{p}}\right)^{2}=1-(1-\eta)^{2},
$$

where $E$ is the elasticity modulus before blasting, $c_{p}$ is the wave velocity before blasting, $\bar{E}$ is the equivalent elasticity modulus after blasting, $\bar{c}_{p}$ is the wave velocity after blasting, and $\eta$ is the reduction rate of the wave velocity.

In the strict sense, $D>0$ represents the fact that rock mass is damaged. However, considering the influence of blasting damage on the properties of rock mass, the code SL47-94 stipulates that rock mass can be affected by blasting damage when $\eta>10 \%$ and the corresponding damage threshold is 0.19 .

2.2. Partition of Damage Zone. Generally, due to strong impact, rock mass blasting with the coupled charge or smaller decoupling coefficient charge forms the crushed zone, the fracture zone, and the vibration zone with the borehole as the center. In engineering, the decoupled charge is mostly used to weaken shock effect and eliminate the crushed zone so as to reduce the blasting effect on the reserved rock mass. That is to say, the purpose is to avoid producing the serious damage zone of "cracking without breaking" from the perspective of damage. The decoupled charge structure usually contains the radial and axial decoupled charge structure, so applying the volumetric decoupling coefficient can describe the radial and axial decoupling coefficients. The smooth blasting technology is used in the perimeter controlled blasting. The radial and axial decoupled charge structure is usually adopted in the borehole. Particularly, it is more likely to form local fracture and damage around the charge segment. It is obvious that the influence of radial decoupling coefficients around the charge segment on the radial distribution laws of damage factors should be studied in advance or emphatically under a certain volumetric decoupling coefficient. For this purpose, the maximum radial decoupling coefficient of "cracking without breaking" caused by rock mass blasting, $K_{0}$, is defined as the critical radial decoupling coefficient whose value is related with properties of rock mass and explosive. When the radial decoupling coefficient is greater than $K_{0}$, namely, $K \geq K_{0}$, rock mass around the borehole can be partitioned into three zones: the general damage zone, the slight damage zone, and the undamaged zone in turn, as shown in Figure 1(a). When $K<K_{0}$, rock mass around the borehole can be partitioned into four zones: the serious damage zone, the general damage zone, the slight damage zone, and the undamaged zone in turn, as shown in Figure 1(b).

The abovementioned rock mass damage in each zone, which represents growth or coalescence of microcracks, results from the combined action of explosion stress wave and quasistatic gas. The characteristics of rock mass damage in each zone can be defined. In the serious damage zone, rock mass discontinuities and rock blocks are all fractured, but do not fully form intersecting blocks; that is, blasting cracks in rock mass have not been fully coalesced. In the general damage zone, rock mass discontinuities are fractured while rock blocks are not; that is, microcracks in rock blocks have not been grown to form blasting cracks. In the slight damage zone, rock mass discontinuities are locally fractured, but growth of fractured cracks is extremely limited and microcracks in rock blocks are not grown. In the undamaged zone, pressure decreases with the increase of distance from the borehole and is not enough to make microcracks grow, so rock mass is not damaged. 


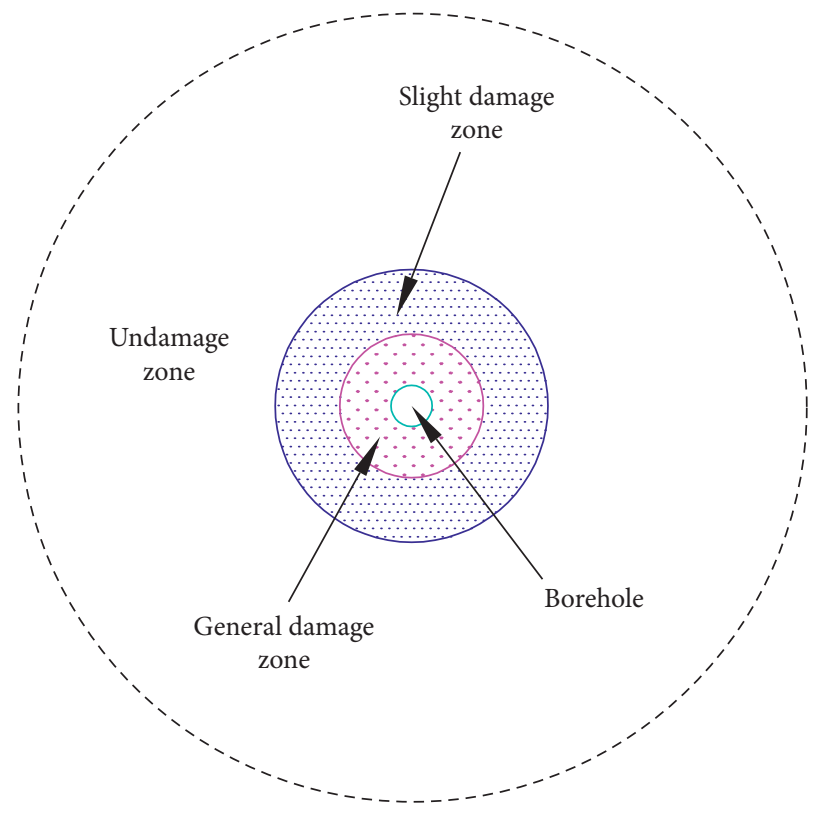

(a)

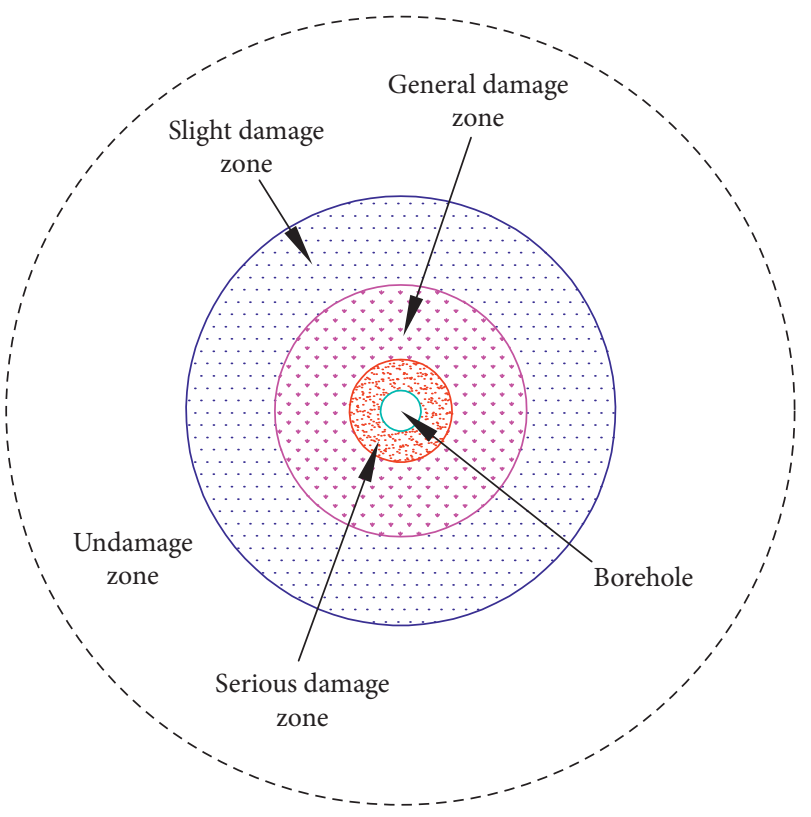

(b)

Figure 1: Partition of damage zone under radial decoupled charge blasting. (a) $K \geq K_{0}$. (b) $K<K_{0}$.

\section{Distribution Laws of Damage Factors}

3.1. Analysis and Assumptions. In general, there exist two different actions under the blasting load, that is, the action of explosion stress wave and the action of quasistatic gas, and both cause different degrees of damage to rock mass. Although the radial and axial decoupled charge structure is used in smooth or presplit blasting, which can weaken the explosion impact greatly, damage caused by the action of explosion stress wave can provide favorable conditions for the action of quasistatic gas so that damage caused by the action of quasistatic gas cannot be ignored. Therefore, it is necessary to study the radial distribution laws of damage factors under the combined action of the two.

The abovementioned action processes are sequential but complex, so it is difficult to separate them completely. To simplify the analysis, when deriving the analytical solution of damage factors under the combined action of the two, the action of explosion stress wave and quasistatic gas should be considered separately in advance and then be superposed together. Besides, the blasting damage is a function of time and space and it is complex to consider the continuous time effect when studying the radial distribution laws of damage factors. Therefore, the distribution laws of the maximum damage factor in two discrete time points selected from two periods of explosion stress wave and quasistatic gas are only focused on. Pressure of time points acted by explosion stress wave takes the stress peak value and that acted by quasistatic gas takes the quasistatic pressure value.

In smooth or presplit blasting, the cracks grow directionally along the connecting line of the borehole centers and the growth of cracks in surrounding rock is restrained due to the stress concentration effect between the adjacent holes. The stress concentration zone along the connecting line of the borehole centers is relatively smaller than the damage zone of surrounding rock, so the effect on damage of surrounding rock can be ignored. In addition, the damage characteristics in surrounding rock are generally focused on in smooth blasting. The stress concentration effect under the influence of adjacent holes is mainly on the connecting line of the borehole centers, while the influence of the stress concentration effect on surrounding rock is less, especially on the right back of boreholes. Therefore, smooth or presplit blasting can be simplified as single-hole blasting for analytical analysis.

It is easy to obtain initial parameters of homogeneous material before blasting, while the change laws of material parameters after blasting is unclear. And for a mass point, the wave velocity changes with time after blasting. Because the propagation velocity of stress wave is faster than the growth velocity of microcracks and wave velocity in the equation $\sigma=\rho_{r} c v$ is still the original wave velocity before blasting, $c_{p}$, it is difficult to derive the relation between wave velocity and stress after blasting by using this equation. The essence of material damage is nucleation, growth, and connection of microcracks, while the mechanical description of microcracks is exactly made by mesodamage mechanics to reveal the essence of material damage. On the one hand, mesodamage mechanics ignores the microscopic physical process in which damage is too complex and avoids the heavy calculation of statistical mechanics. On the other hand, it contains geometrical and physical characteristics of mesodamage of different materials and provides a clear physical background for the damage variables and the evolution process of damage. Besides, the average idea is adopted in mesodamage mechanics and the results of study on damage mechanism of mesoscopic structure are reflected on the behavior description of macromechanics [28, 29]. 
Therefore, the interrelation between microcracks and the radial distribution laws of damage factors can be communicated from the perspective of effective elastic modulus of mesodamage mechanics.

The explosive is usually in the center of a borehole. Detonation gas expands around the explosive with the same high speed and acts on the borehole wall. In addition, rock mass is generally in the state of initial ground stress which is very small relative to the blasting load. Meanwhile, rock mass under the blasting load shows a quasibrittle characteristic. For smooth blasting holes, the amplitude value of explosive stress around the charge segment is the largest and reduces gradually in the process of the axial transmission to the air segment. When the borehole is filled with detonation gas, quasistatic pressure is finally formed so as to make damage around the charge segment greater than that around the air interval segment [24]. Therefore, from the most unfavorable perspective, the radial distribution laws of damage factors within the range of the charge segment under decoupled charge blasting are only considered when the linear charge density is fixed. To highlight the focus and simplify the analysis, the following assumptions are made: (1) The influence of initial ground stress is not taken into account. (2) Distribution of borehole pressure is uniform. (3) Rock mass is elastic material containing microcracks with uniformly random distribution. (4) The action of explosion stress wave and quasistatic gas are considered separately.

3.2. Derivation of Theoretical Formulas. According to the theory of mesodamage mechanics, the calculation methods of the effective elastic modulus mainly include Taylor's method without considering the interaction among microcracks, the methods considering the weak interaction among microcracks, such as the self-consistent method, the generalized self-consistent method, Mori-Tanaka's method, and the differential method, and the statistical mesomechanics method considering the strong interaction among microcracks. For most quasibrittle materials, the influence of the interaction among microcracks on the constitutive relation is not obvious, and meanwhile, there is no simple and effective method to calculate the interaction effect among microcracks $[28,29]$. Therefore, this paper uses the relatively simple Taylor method to analyze rock mass with microcracks having the same size and uniformly random distribution of orientation and location. The effective elastic modulus of rock mass with microcracks is [8]

$$
\frac{\bar{E}}{E}=\left(1+A N a^{m}\right)^{-1},
$$

where $\bar{E}$ is the effective elastic modulus, $E$ is the initial elastic modulus, $N$ is the number of microcracks in per unit volume, and $a$ is the average radius of activated microcracks. For the three-dimensional problem, $A=16\left(1-\mu^{2}\right)$ $(10-3 \mu) /(45(2-\mu))$, where $\mu$ is the Poisson ratio and $m=3$. For the two-dimensional problem, $A=\pi$ and $m=2$.

The average radius of microcracks in rock mass can be calculated by Grady's expression [30]:

$$
a=\frac{1}{2}\left(\frac{\sqrt{20} K_{I C}}{\rho_{r} c_{p} \dot{\varepsilon}_{\max }}\right)^{2 / 3},
$$

where $K_{I C}$ is the fracture toughness, $\rho_{r}$ is the rock mass density, $c_{p}$ is the $\mathrm{P}$-wave velocity, and $\dot{\varepsilon}_{\text {max }}$ is the maximum of the volumetric strain rate.

The abovementioned effective elastic modulus is the homogenization modulus value based on the statistical law. It can be approximately applied to the change laws of the average modulus in an annulus formed by the microelements with the distance of $r$ from the borehole center; namely, $\bar{E}(r)=f(N(r))$.

The number of microcracks caused by blasting is directly proportional to the peak value of explosion stress wave [31]; namely,

$$
N(r)=k_{d} \sigma_{r d}=k_{d} p_{0 d}(\bar{r})^{-\alpha}=k_{d} p_{0 d}\left(\frac{r}{r_{b}}\right)^{-\alpha},
$$

where $k_{d}$ is the proportional coefficient under the action of explosion stress wave, $p_{0 d}$ is the peak value of pressure on the borehole wall under the action of explosion stress wave, $r_{b}$ is the borehole radius, $\bar{r}$ is the scaled distance, $r$ is the distance from the borehole center, and $\alpha$ is the attenuation index. For the intact rock mass, $\alpha=2-\mu_{d} /\left(1-\mu_{d}\right)$, where $\mu_{d}$ is the dynamic Poisson's ratio, while for the fractured rock mass, the value may be greater than 2.0.

Thus, the damage factor under the action of explosion stress wave can be obtained from

$$
D_{d}(r)=1-\left(1+A_{d} k_{d} a^{m} p_{0 d}\left(\frac{r}{r_{b}}\right)^{-\alpha}\right)^{-1},
$$

where for the three-dimensional problem, $A_{d}=16\left(1-\mu_{d}^{2}\right)$ $\left(10-3 \mu_{d}\right) /\left(45\left(2-\mu_{d}\right)\right)$ and for the two-dimensional problem, $A_{d}=\pi$.

Based on the thick-walled cylinder theory, the stress change law under the action of quasistatic gas is

$$
\sigma_{r j}=p_{0 j}(\bar{r})^{-2}
$$

where $p_{0 j}$ is the pressure on the borehole wall under the action of quasistatic gas.

It is assumed that the average radius of microcracks is invariable. In addition, Poisson's ratio and the number of activated microcracks under explosion stress wave are different from those under quasistatic gas, so $A$ and $k$ also change. Thus, the damage factor under the action of quasistatic gas can be obtained from

$$
D_{j}(r)=1-\left(1+A_{j} k_{j} a^{m} p_{0 j}\left(\frac{r}{r_{b}}\right)^{-2}\right)^{-1},
$$

where $k_{j}$ is the proportional coefficient under the action of quasistatic gas. For the three-dimensional problem, $A_{j}=16\left(1-\mu_{j}^{2}\right)\left(10-3 \mu_{j}\right) /\left(45\left(2-\mu_{j}\right)\right)$, where $\mu_{j}$ is the static Poisson's ratio. For the two-dimensional problem, $A_{j}=\pi$.

Blasting damage is the result of the combined action of explosion stress wave and quasistatic gas, but their 
contributions to damage are different at different stages. In fact, the two actions intertwine and couple with each other. Therefore, the damage factor under the combined action of the two can be expressed by [27]

$$
D(r)=1-\left(1-D_{d}(r)\right) \times\left(1-D_{j}(r)\right) \text {. }
$$

In conclusion, with the consideration of the combined action of explosion stress wave and quasistatic gas, the damage factor around a borehole is

$$
\begin{aligned}
D(r)= & 1-\left(1+A_{d} k_{d} a^{m} p_{0 d}\left(\frac{r}{r_{b}}\right)^{-\alpha}\right)^{-1} \\
& \times\left(1+A_{j} k_{j} a^{m} p_{0 j}\left(\frac{r}{r_{b}}\right)^{-2}\right)^{-1},
\end{aligned}
$$

where the proportional coefficients, $k_{d}$ and $k_{j}$, can be determined by experiments or analogy methods and the pressure on the borehole wall under decoupled charge, $p_{0 d}$ and $p_{0 j}$, can be determined according to the expansion laws of detonation gas and detonation pressure.

In particular, for the radial decoupled and axial continuous charge structure, the detonation pressure takes an average; namely, $p_{m}=1 / 8 \rho_{e} D_{m}^{2}$, where $\rho_{e}$ is the explosive density and $D_{m}$ is the detonation velocity. Rock mass can be dealt with as a plane strain problem. For the radial decoupled and axial interval charge structure, the detonation pressure is $p_{m}=1 / 4 \rho_{e} D_{m}^{2}$. Rock mass near the charge segment is constrained sideward by rock mass near the air segment and when simplified as a plane stress problem, there are some discrepancies with the reality, so it is more practical to consider it as a three-dimensional problem. Pressure on the borehole wall under explosion stress wave usually adopts the quasistatic pressure to multiply by the pressure enhancement coefficient which is determined by the radial decoupling coefficient values. When the radial decoupling coefficient is less than the critical value, the blasting effect belongs to the strong impact and the bigger pressure enhancement coefficient is chosen. Conversely, it belongs to the weak impact and the smaller pressure enhancement coefficient is chosen. In general, the pressure enhancement coefficients can be taken from 2 to 11 .

Through analysis, it is found that the broad trend of distribution curves under the radial decoupled charge is basically close, but the change rate of distribution curves and the maximum of damage factors are different so as to cause different damage ranges. A comparison of the distribution laws of damage factors under three decoupling coefficients is made. The material parameters are given in the second row of Table 1 , and blasting parameters are given in the second row of Table 2. The curves of radial distribution laws are shown in Figure 2.

Damage factors under decoupled charge blasting decrease in a power function and finally tend to zero with the increase of distance from the center of the borehole, as shown in Figure 2. This is mainly because the action of explosion stress wave is prominent at the initial stage and the contribution to the blasting damage is greater. With the decrease of explosion stress wave, the action of quasistatic gas is increasingly prominent. The theoretical formula gives full consideration to the combined action of explosion stress wave and quasistatic gas, which can characterize the radial distribution laws of damage factors and reflect the action process of rock mass blasting.

The attenuation rate of damage factors decreases gradually when $K \geq K_{0}$ and firstly increases and then decreases when $K<K_{0}$, as shown in Figure 2. The main reason is that damage caused by explosion stress wave is smaller and the conditions offered for the action of quasistatic gas are limited when $K \geq K_{0}$, while damage caused by explosion stress wave is larger and the conditions offered for the action of quasistatic gas are superior when $K<K_{0}$.

In Figure 2, when $K \geq K_{0}$, the explosion impact is greatly weakened and the serious damage zone is not generally produced. A serious damage zone whose largest boundary is located in the first turning point of the distribution curve is generated when $K<K_{0}$. With other things being equal, the damage range decreases gradually with the increase of radial decoupling coefficients. When 0.19 is taken as a damage threshold value, the damage range of the decoupling coefficient of 1.31 is about 3.57 times that of the decoupling coefficient of 1.68. The difference of the damage range is related with explosive properties, charge structures and rock mass properties, and so on.

From the perspective of the size of the damage range and whether the serious damage zone is produced or not, the appropriate radial decoupling coefficient which is greater than the critical value is chosen in the field charge structure by explosive parameters and rock mass properties to protect the integrity and stability of surrounding rock.

\section{Experimental Verification and Analysis}

4.1. Model Experiment. Two cylinder models numbered A and $\mathrm{B}$, respectively, are poured by using fine stone concrete. The diameter is $100 \mathrm{~cm}$ and the height is $50 \mathrm{~cm}$. The curing period under the natural condition is more than 28 days. Meanwhile, the cubic standard samples of $15 \mathrm{~cm}$ are made. After curing, the physical and mechanical parameters are tested, which are listed in the third row of Table 1 . It is observed that this concrete is equivalent to rock mass with low strength. A borehole in the center of the cylinder is drilled, as shown in Figure 3. The blasting parameters are listed in the third row of Table 2.

In the process of the experiments, the models are erected to test P-wave velocity of concrete by applying the opposite test method so as to avoid the second disturbance and the influence of water injection during the process of the coring test. Besides, damage tested by the opposite test method contains the comprehensive damage around the air and charge segment, which can test the maximum degree and range of damage. It is shown from the distribution characteristics of pressure in the borehole that damage around the charge segment is more serious than that around the air segment. Therefore, damage around the charge segment is often a key concern and the opposite test results can reflect damage around the charge segment, which shows that the 
TABLe 1: Physical and mechanical parameters of materials in different applied cases.

\begin{tabular}{lccccccc}
\hline Cases & $\rho_{r}\left(\mathrm{~kg} / \mathrm{m}^{3}\right)$ & $E(\mathrm{GPa})$ & $\mu_{j}$ & $c_{p}(\mathrm{~m} / \mathrm{s})$ & Uniaxial compressive strength $\sigma_{u}(\mathrm{MPa})$ & $K_{I C}\left(\mathrm{MPa} \cdot \mathrm{m}^{1 / 2}\right)$ & $\dot{\varepsilon}_{\text {max }}$ \\
\hline Theoretical calculation & 2600 & - & 0.30 & 4000 & 50.0 & 0.9 & $10^{3}$ \\
Model experiment & 2356 & 36.68 & 0.27 & 4457 & 37.2 & - & - \\
Model experiment [24] & 2000 & - & - & 3580 & 20.0 & - \\
\hline
\end{tabular}

TABLE 2: Blasting parameters in different applied cases.

\begin{tabular}{|c|c|c|c|c|c|c|c|c|}
\hline Cases & $\begin{array}{c}\text { Borehole } \\
\text { diameter } d_{b}(\mathrm{~mm})\end{array}$ & $\begin{array}{c}\text { Borehole } \\
\text { depth } l_{b}(\mathrm{~cm})\end{array}$ & $\begin{array}{c}\text { Charge } \\
\text { length } l_{c}(\mathrm{~cm})\end{array}$ & $\begin{array}{c}\text { Stemming } \\
\text { length } l_{d}(\mathrm{~cm})\end{array}$ & K & $\rho_{e}\left(\mathrm{~g} / \mathrm{cm}^{3}\right)$ & $D_{m}(\mathrm{~m} / \mathrm{s})$ & $K_{0}$ \\
\hline Theoretical calculation & 42 & - & - & - & 1.31 and 1.68 & 1.0 & 3200 & 1.43 \\
\hline Model experiment & 40 & 30 & 2 and 5 & 20 & 1.25 and 2.0 & 1.0 & 3200 & 1.55 \\
\hline Model experiment [24] & - & 10 & 3 & 5 & 2.0 & - & - & - \\
\hline
\end{tabular}

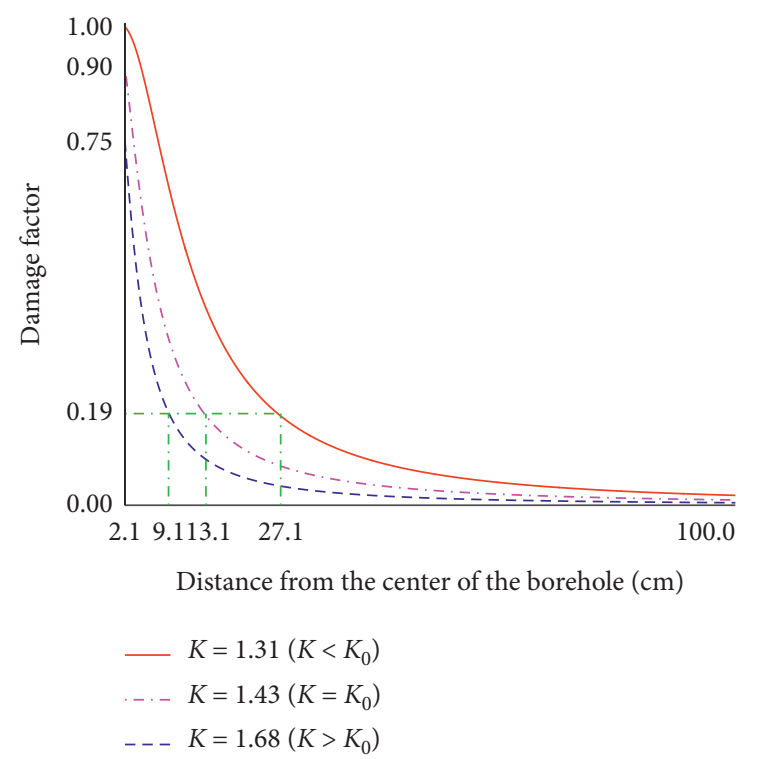

Figure 2: Comparison of distribution curves of damage factors under different radial decoupling coefficients.

test method is reasonable and feasible. Four mutually vertical P-wave test lines, named successively from I to IV in a clockwise direction, are laid out on the surface of the models. There are eight test points in each line to verify each other, and then the average of $\mathrm{P}$-wave velocity is taken to reduce the error caused by randomness of concrete properties. The area close to the borehole is densely arranged to reflect the trend of damage change obviously, while the area far away from the borehole is sparsely arranged. Therefore, considering the radius of the concrete models and the radius of the test probes, the distance of the test points from the center of the borehole are $4 \mathrm{~cm}, 6 \mathrm{~cm}, 8 \mathrm{~cm}, 12 \mathrm{~cm}, 17 \mathrm{~cm}, 23 \mathrm{~cm}$, $31 \mathrm{~cm}$, and $40 \mathrm{~cm}$ in turn, as shown in Figure 3.

In the models numbered $\mathrm{A}$ and $\mathrm{B}$, the radial decoupling coefficients of 1.25 and 2.0 are, respectively, used to carry out the blasting experiments. The explosive is No. 2 rock emulsion explosive with the charge amount of $15 \mathrm{~g}$, which are detonated by an instantaneous electric detonator, as shown in Figures 4(a) and 4(b). The sandy clay is used to

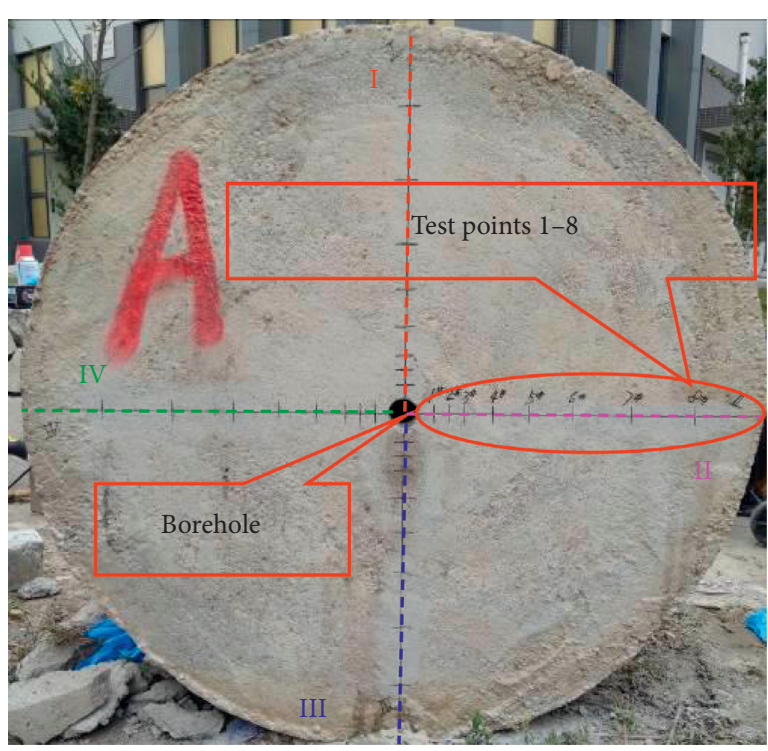

FIgURE 3: Fine stone concrete model and test lines of P-wave velocity.

stem the borehole, as shown in Figure 4(c). There are some axial air intervals near the charge segment. The condition after blasting is shown in Figure 4(d). And then the P-wave velocity of the models is tested by using the opposite test method and the RSM-SY5(T) sonic detector, as demonstrated in Figure 4(e). Before testing the P-wave velocity of the models, firstly, the area of the test points is polished to smoothness. Secondly, auxiliary circles with the same diameter as the probe are drawn with the test points as the center to ensure that the transmitting probe and the receiving probe are in alignment. Thirdly, the coupling agent is used to make the probe fit the concrete surface closely. The tested curves of P-wave are displayed in Figure 5.

The damage factors are calculated by bringing the tested results of $\mathrm{P}$-wave velocity into (1). In addition, the coefficient $A$, the average radius of cracks, and the borehole pressure are calculated with the parameters in Tables 1 and 2. The proportional coefficient, $k$, is fitted by the blasting experiments. The theoretical curves are calculated by bringing the 


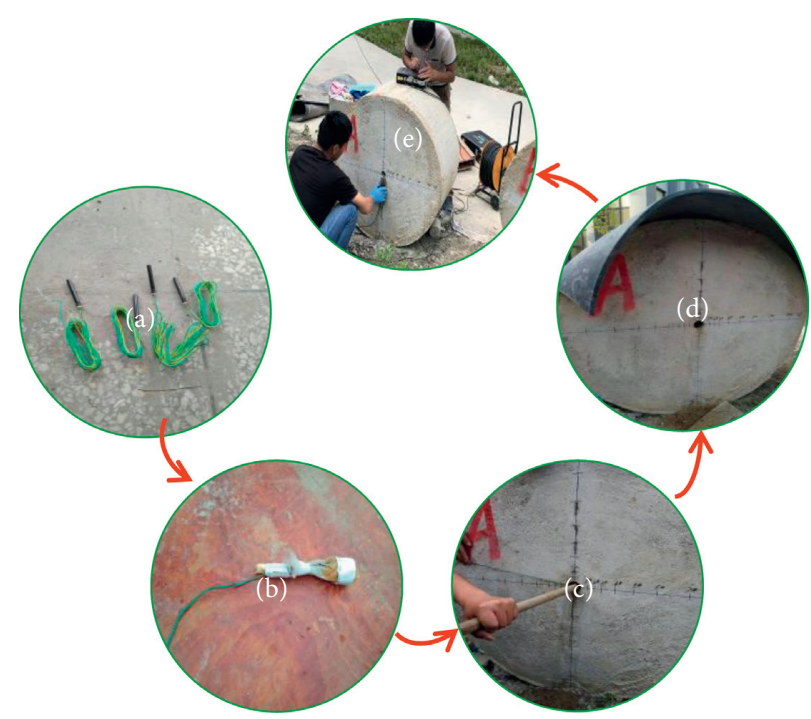

Figure 4: Process of blasting experiment. (a) Electric detonator. (b) Explosive cartridge. (c) Borehole stemming. (d) After blasting. (e) P-wave testing.

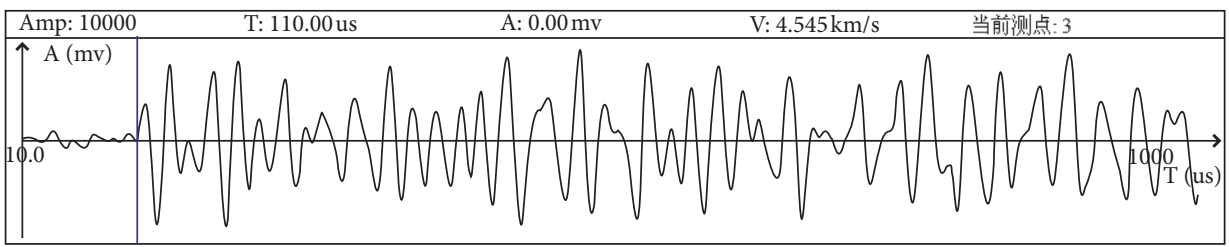

(a)

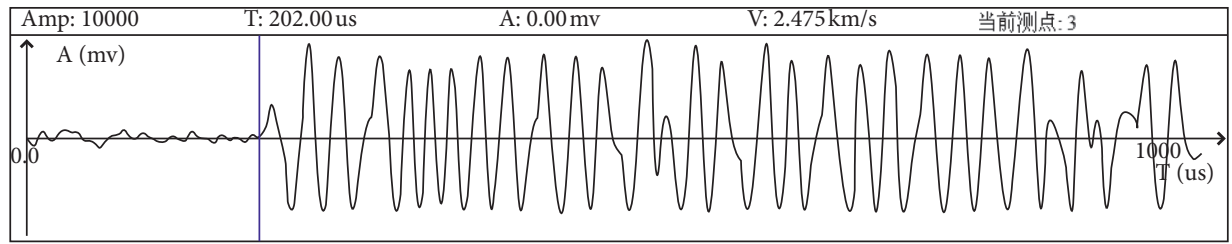

(b)

Figure 5: The P-wave test curves of test point No. 3 of test line I under $K=1.25$. (a) Before blasting. (b) After blasting.

abovementioned parameters into (9). The tested values and the calculated curves of damage factors under the two radial decoupling coefficients are shown in Figure 6.

In Figure 6, the test values of damage factors under the two radial decoupling coefficients decrease nonlinearly with the increase of the distance from the center of the borehole. When the radial decoupling coefficient is larger, the attenuation rate is faster and the maximum damage factor and the damage range around the borehole are smaller. Conversely, due to the appearance of the serious damage zone under the smaller radial decoupling coefficients, the attenuation rate is slower and the maximum damage factor and the damage range around the borehole are larger.

The radial decoupling coefficient has a great influence on the damage range. When 0.19 is taken as a damage threshold value, the damage range of the decoupling coefficient of 1.25 is about 5 times that of the decoupling coefficient of 2.0, as shown in Figure 6. Thus, it is necessary to choose an appropriate radial decoupling coefficient for controlling the damage range from the perspective of the charge structure.

The theoretical formula reflecting the combined action of explosion stress wave and quasistatic gas is adopted to calculate damage factors based on the model parameters. It is found that the correlation is high and the theoretical formula can describe the radial distribution laws of damage factors around the borehole so as to estimate the damage range, as displayed in Figure 6. Besides, when the radial decoupling coefficients change, it can also reflect the influence of the load characteristics in the borehole on damage; namely, when the radial decoupling coefficient is larger than the critical value, the impact effect of the blasting load on the borehole wall is greatly weakened.

4.2. Cited Model Experiment. In the experiment [24], cement mortar models, whose cement-sand ratio was designed as $1: 2.5$ and water-cement ratio was 0.4 , were made. The physical and mechanical parameters are presented in the fourth row of 


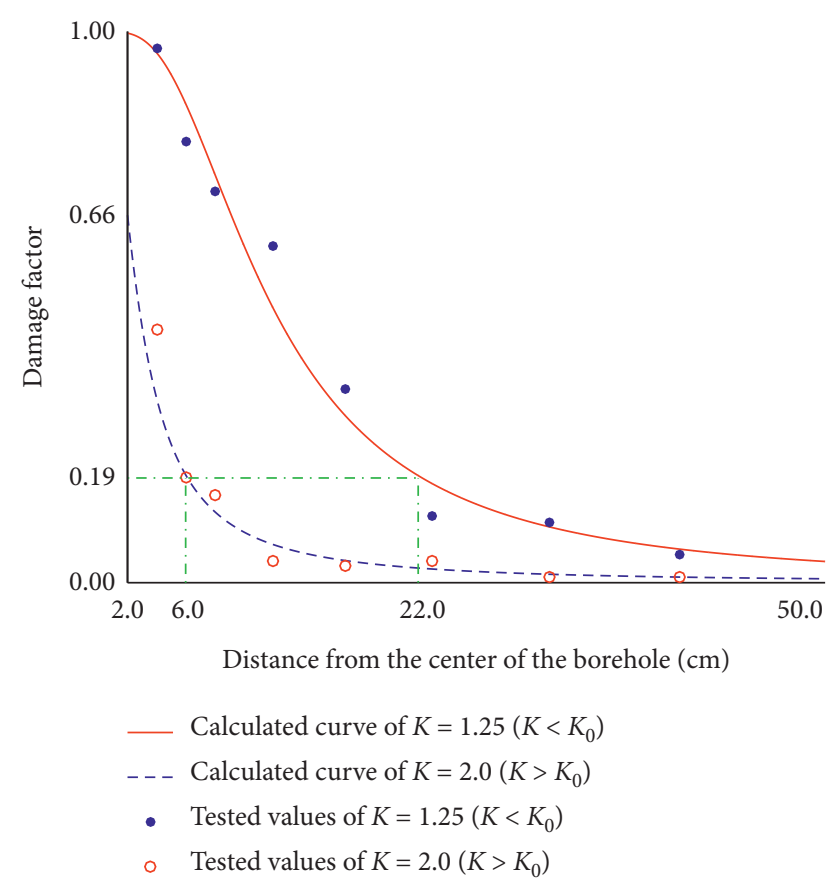

Figure 6: Comparison and verification of theoretical curves and test values of damage factors under different radial decoupling coefficients.

Table 1. The single-hole blasting is carried out in the models, and the blasting parameters adopted are listed in the fourth row of Table 2. The fitting relation between damage factors and the scaled distance is shown by the dashed line in Figure 7. A curve is obtained from the theoretical formula derived in this paper, as shown by the solid line in Figure 7.

On the basis that stress and strain around the borehole decrease in a power function with the increase of distance from the blasting source, Dai et al. [24] used the power function, $D(r)=\bar{A}\left(r / r_{b}\right)^{-\bar{B}}$, where $\bar{A}$ and $\bar{B}$ reflect coefficients, such as rock mass properties, charge structure, detonation conditions, and so on, to fit out a quantitative relation which has a good consistency with the change laws of stress and strain in (4).

Obtained from the derived theoretical formula in this paper which can reflect the combined action of explosion stress wave and quasistatic gas, the radial distribution laws of damage factors have a good correlation with the tested values, as shown in Figure 7. Meanwhile, the correlation coefficient is higher than that fitted by a power function because the attenuation index under the action of explosion stress wave is different from that under the action of quasistatic gas. The same attenuation index was used in [24], while the two different attenuation indexes are considered in the theoretical formula derived in this paper.

It is thought that the attenuation laws of damage factors have a good consistency with the change laws of stress and strain [24]. Damage of rock mass is the result of the actions of explosion stress and strain from the aspect of mechanical mechanism. Based on the effective elastic modulus and the change law of stress, this paper derives a theoretical formula

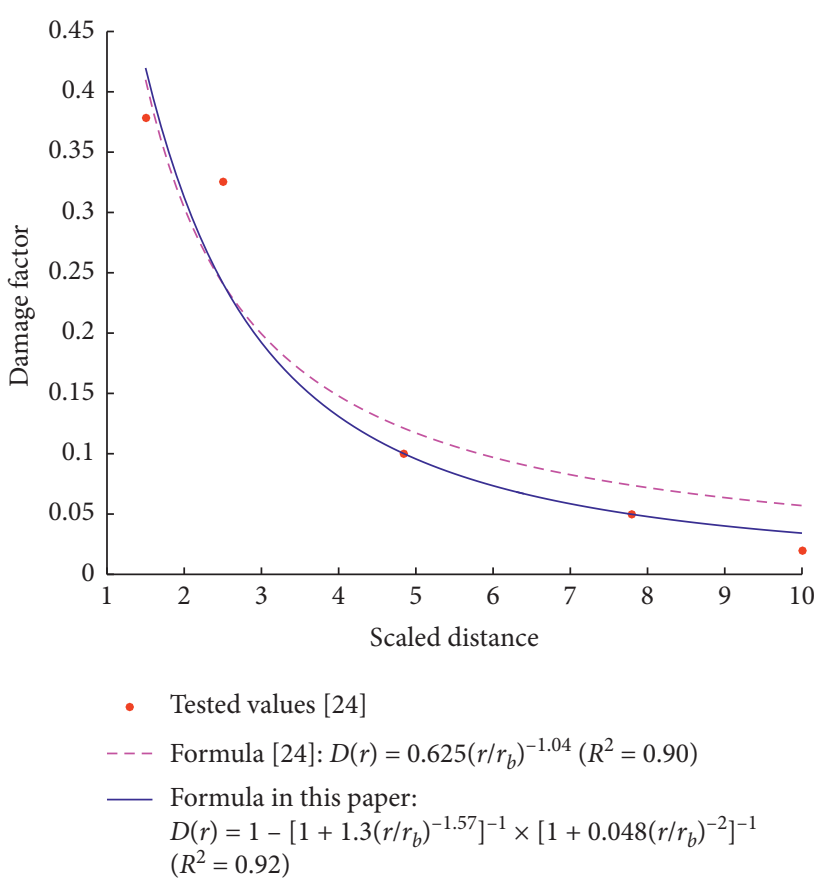

FIgure 7: Comparison and verification of theoretical formula curve, tested values [24], and empirical formula curve [24] of damage factors under decoupled charge blasting.

under the combined action of explosion stress wave and quasistatic gas. The formula can describe the distribution laws of damage factors and estimate the damage range, which can not only provide an important reference for studying the damage characteristics, but also reveal the blasting damage mechanism. Therefore, the formula has a great theoretical significance.

\section{Conclusion}

The formula of the radial distribution laws of damage factors caused by decoupled charge blasting under the combined action of explosion stress wave and quasistatic gas is derived and verified. The following conclusions are drawn:

(1) The derived formula can describe the radial distribution laws of damage factors, embody the influence of the radial decoupling coefficients on damage factors, and communicate the interrelation between damage factors and blasting parameters. Meanwhile, the formula is of high accuracy and wide practicability. It can be conveniently applied to engineering to guide the optimization design of blasting parameters

(2) The attenuation rate of damage factors decreases gradually when $K \geq K_{0}$ and first increases and then decreases when $K<K_{0}$. In addition, the attenuation rate is in positive correlation with the radial decoupling coefficients at the initial stage. But with other things being equal, the damage range is in negative correlation with the radial decoupling coefficients 
(3) The formula of the radial distribution laws of damage factors is derived from the perspective of mechanics. Parameters in the formula have definite physical meanings. And the formula has a relatively simple form and a strict theoretical basis. Besides, the mechanical mechanism of blasting damage can be revealed. Therefore, it can enrich the damage theory of rock mass blasting

(4) In engineering, an appropriate radial decoupling coefficient which is larger than the critical value should be adopted to the charge structure of smooth or presplit blasting on the premise of stable detonation so as to reduce the disturbance of the blasting action to surrounding rock and protect the integrity and stability of rock mass.

\section{Data Availability}

The data used to support the findings of this study are available from the corresponding author upon request.

\section{Conflicts of Interest}

The authors declare that there are no conflicts of interest regarding the publication of this paper.

\section{Acknowledgments}

This work was financially supported by the National Natural Science Foundation of China (Grant no. 50574076).

\section{References}

[1] Z. Fan, J. Zhang, H. Xu, and J. Cai, “Transmission of normal P-waves across a single joint based on $\mathrm{g}-\lambda$ model," Shock and Vibration, vol. 2019, Article ID 8240586, 10 pages, 2019.

[2] D. Xiao, Z. Zhu, R. Hu, and L. Lang, "Study of testing method for dynamic initiation toughness of sandstone under blasting loading," Shock and Vibration, vol. 2018, Article ID 1043298, 13 pages, 2018.

[3] A. Daehnke, H. P. Rossmanith, and R. E. Knasmillner, "Blast induced dynamic fracture propagation," in Proceedings of the 5th International Symposium on Rock Fragmentation by Blasting, pp. 13-24, Montreal, Canada, August 1996.

[4] L. Liu and P. D. Katsabanis, "Development of a continuum damage model for blasting analysis," International Journal of Rock Mechanics and Mining Sciences, vol. 34, no. 2, pp. 217-231, 1997.

[5] W.-B. Lu, Y.-G. Hu, J.-H. Yang, M. Chen, and P. Yan, "Spatial distribution of excavation induced damage zone of high rock slope," International Journal of Rock Mechanics and Mining Sciences, vol. 64, no. 6, pp. 181-191, 2013.

[6] S. Kwon, C. S. Lee, S. J. Cho, S. W. Jeon, and W. J. Cho, “An investigation of the excavation damaged zone at the kaeri underground research tunnel," Tunnelling and Underground Space Technology, vol. 24, no. 1, pp. 1-13, 2009.

[7] H. B. Li, X. Xia, J. C. Li, and J. Zhao, "Rock damage control in bedrock blasting excavation for a nuclear power plant," International Journal of Rock Mechanics and Mining Sciences, vol. 48, no. 2, pp. 210-218, 2011.

[8] L. M. Taylor, E.-P. Chen, and J. S. Kuszmaul, "Microcrackinduced damage accumulation in brittle rock under dynamic loading," Computer Methods in Applied Mechanics and Engineering, vol. 55, no. 3, pp. 301-320, 1986.

[9] R. Yang, W. F. Bawden, and P. D. Katsabanis, "A new constitutive model for blast damage," International Journal of Rock Mechanics and Mining Sciences \& Geomechanics Abstracts, vol. 33, no. 3, pp. 245-254, 1996.

[10] M. Yazdchi, S. Valliappan, and W. Zhang, "A continuum model for dynamic damage evolution of anisotropic brittle materials," International Journal for Numerical Methods in Engineering, vol. 39, no. 9, pp. 1555-1583, 1996.

[11] L. R. Johnson and C. G. Sammis, "Effects of rock damage on seismic waves generated by explosions," Monitoring the Comprehensive Nuclear-Test-Ban Treaty: Source Processes and Explosion Yield Estimation, vol. 158, no. 11, pp. 1869-1908, 2001.

[12] S. Esen, I. Onederra, and H. A. Bilgin, "Modelling the size of the crushed zone around a blasthole," International Journal of Rock Mechanics and Mining Sciences, vol. 40, no. 4, pp. 485-495, 2003.

[13] S. K. Mandal, M. M. Singh, and S. Dasgupta, "Theoretical concept to understand plan and design smooth blasting pattern," Geotechnical and Geological Engineering, vol. 26, no. 4, pp. 399-416, 2008.

[14] A. Mitelman and D. Elmo, "Modelling of blast-induced damage in tunnels using a hybrid finite-discrete numerical approach," Journal of Rock Mechanics and Geotechnical Engineering, vol. 6, no. 6, pp. 565-573, 2014.

[15] M. Sagong, C. Soo Park, B. Lee, and B.-S. Chun, "Cross-hole seismic technique for assessing in situ rock mass conditions around a tunnel," International Journal of Rock Mechanics and Mining Sciences, vol. 53, pp. 86-93, 2012.

[16] M. Ramulu, A. K. Chakraborty, and T. G. Sitharam, "Damage assessment of basaltic rock mass due to repeated blasting in a railway tunnelling project-a case study," Tunnelling and Underground Space Technology, vol. 24, no. 2, pp. 208-221, 2009.

[17] H. Chu, X. Yang, S. Li, and W. Liang, "Experimental investigation of the propagation and attenuation rule of blasting vibration wave parameters based on the damage accumulation effect," Shock and Vibration, vol. 2018, Article ID 2493149, 9 pages, 2018.

[18] E. Hamdi, N. B. Romdhane, and J. M. Le Cléac'h, “A tensile damage model for rocks: application to blast induced damage assessment," Computers and Geotechnics, vol. 38, no. 2, pp. 133-141, 2011.

[19] H. Fattahi, S. Shojaee, M. A. E. Farsangi, and H. Mansouri, "Hybrid Monte Carlo simulation and ANFIS-subtractive clustering method for reliability analysis of the excavation damaged zone in underground spaces," Computers and Geotechnics, vol. 54, pp. 201-221, 2013.

[20] M. B. Étkin, A. E. Azarkovich, A. A. Sapronov, and V. G. Vartanov, "Protecting external rock bodies from blasting damage," Hydrotechnical Construction, vol. 35, no. 9, pp. 499-506, 2001.

[21] S. S. Rathore and S. Bhandari, "Controlled fracture growth by blasting while protecting damages to remaining rock," Rock Mechanics and Rock Engineering, vol. 40, no. 3, pp. 317-326, 2007.

[22] A. Tamai, H. Shirahata, and K. Nagahisa, "Effect of controlled blasting and the evaluation of the influence on rock mass around tunnel," Journal of Tunnel Engineering Jsce, vol. 2, pp. 209-214, 2011.

[23] H. Hu, W. Lu, P. Yan, M. Chen, and Q. Gao, "A vibrationisolating blast technique with shock-reflection device for dam 
foundation excavation in complicated geological conditions," Shock and Vibration, vol. 2018, Article ID 8029513, 11 pages, 2018.

[24] J. Dai, Y. L. Wan, and C. L. Xu, "Experimental study on damage to surrounding rock mass by contour blasting," Chinese Journal of Rock Mechanics and Engineering, vol. 24, no. 1, pp. 4728-4734, 2005.

[25] Y. L. Suo, "Study for macro-damage degree of weakening blast in hard top-coal," Rock and Soil Mechanics, vol. 26, no. 6, pp. 893-895, 2005.

[26] H. M. Tang, Y. T. Zhou, and Y. P. Liao, "Damage zone of surrounding rock of underground engineering under construction blasting," Journal of Vibration and Shock, vol. 34, no. 23, pp. 202-206, 2015.

[27] P. F. Pan, H. G. Sun, Z. H. Han, C. Feng, R. K. Guo, and S. H. Li, "Numerical study on damage and fracture degree of rock mass induced by blasting mining," Metal Mine, vol. 480, no. 6, pp. 1-7, 2016.

[28] D. Krajcinovic, Damage Mechanics, Elsevier, Amsterdam, Netherlands, 1997.

[29] X. Q. Feng and S. W. Yu, "Estimate of effective elastic moduli with microcrack interaction effects," Theoretical and Applied Fracture Mechanics, vol. 34, no. 3, pp. 225-233, 2000.

[30] D. E. Grady and M. E. Kipp, "Continuum modelling of explosive fracture in oil shale," International Journal of Rock Mechanics and Mining Sciences \& Geomechanics Abstracts, vol. 17, no. 3, pp. 147-157, 1980.

[31] T. N. Hagan and G. Harries, Drilling and Blasting Technology, Mineral Foundation Inc, Adelaide, Australian, 1977. 